\title{
Notas sobre la subsunción de lo urbano al capital financiero: de las geografías de la financiarización a una agenda exploratoria ${ }^{1}$
}

\section{Notes on the subsumption of the urban to financial capital: from the geographies of financialization to an exploratory agenda}

\author{
Luis Daniel Santana Rivas²
}

\begin{abstract}
RESUMEN
Ciertos elementos comunes de las crisis urbanas, nombradas popularmente como 'estaIlidos sociales', tanto en Chile como más recientemente en Colombia, son útiles para hacer una crítica de la categoría de financiarización urbana. En consecuencia, objetivo del artículo es identificar los 'campos ciegos' -vacíos teóricos y áreas de debate irresueltode las discusiones geográficas sobre la financiarización urbana para plantear una agenda teórica de carácter exploratorio que gira en torno a la conceptualización de las formas contemporáneas de 'subsunción' de lo urbano a los procesos de reproducción del capital financiero. A partir de la presentación de diversas propuestas conceptuales derivadas de casos en Suiza, España, Chile o Brasil se identifica un desbalance en el análisis de la financiarización urbana hacia las dinámicas de producción del espacio urbano y más específicamente de reducción de estas últimas a la producción de ambiente construido. Para trascender esa limitación se sugiere el uso de la categoría de formas de subsunción de lo urbano y se esquematizan cuatro tesis a modo de agenda exploratoria.
\end{abstract}

Palabras clave: financiarización, crisis urbana, producción inmobiliaria, subsunción, geografía.

\section{ABSTRACT}

Certain common elements of urban crises, popularly referred to as 'social outbreaks', both in Chile and more recently in Colombia, are useful for critiquing the category of urban financialization. Consequently, the objective of the article is to identify the 'blind fields' -theoretical voids and areas of unresolved debate- of the geographical discussions on urban financialization in order to propose a theoretical agenda of an exploratory nature that revolves around the conceptualization of contemporary forms of 'subsumption' of the urban to the processes of reproduction of financial capital. From the presentation of various conceptual proposals derived from cases in Switzerland, Spain, Chile or Brazil, an imbalance is identified in the analysis of urban financialization towards the dynamics

El artículo hace parte del desarrollo del proyecto de investigación adscrito al Sistema Hermes de la Universidad Nacional de Colombia, No. 50758 "Vivienda, mercado inmobiliario y urbanización capitalista en la región metropolitana de Medellín (1999-2019): entre las lógicas políticas 
of production of urban space and more specifically of reducing the latter to production. built environment. To transcend this limitation, the use of the category of forms of subsumption of the urban is suggested and four theses are outlined as an exploratory agenda.

Keywords: financialization, urban crisis, real estate production, subsumption, geography.

¿Qué tienen en común la Plaza Baquedano ubicada hacia el pericentro oriental de Santiago de Chile y el Monumento de Los Héroes enclavado casi dentro de una estación del sistema masivo de transporte Transmilenio en Bogotá, Colombia? En primera instancia, además de sus estatuas icónicas, hay una similitud en su situación o emplazamiento urbano: el primero es un espacio de borde entre el popular aunque parcialmente gentrificado centro santiaguino y la 'espina burguesa' que se extiende desde el pericentro oriental hacia las laderas de la cordillera de los Andes, combinando barrios de clase alta y las funciones de comando financiero de la potente economía chilena en el célebre y luminoso 'Sanhattan'; el segundo es un espacio que demarca la frontera entre el pericentro norte, el occidente (predominantemente de clases medias) y el rico 'norte' bogotano 'en el cercano barrio del Chicó se presenta el precio del suelo residencial y comercial más costoso de la metrópoli'.

En segundo lugar, ambos han sido escenarios políticos emblemáticos de intensas y multitudinarias protestas de lo que se ha denominado 'estallidos sociales'; primero en Chile durante 2019 y en Colombia con una primera chispa en noviembre de 2019 y una reactivación desde el 27 de abril de 2021, en medio de la aguda crisis de la Covid-19. Aunque son lugares muy representativos para la representación política que han sellado el 'reescalamiento' los estallidos como fenómenos nacionales, estos no son más que la punta del iceberg de unas intrincadas geografías sociales urbanas de espacios de rebelión y represión estatal, así como de contraespacios de repolitización -ollas comunes, asambleas populares, reapropiación de instalaciones estatales- que no se limitan de ninguna manera a las metrópolis capitalinas sino que se expresan con intensidad en otras ciudades regionales -Valparaíso en Chile o Cali en Colombia, por ejemplo-.

En tercer y último lugar, los procesos causales que han dado lugar a esos estallidos tienen una serie raíces comunes, a pesar de las inmensas diferencias de toda índole, entre las realidades chilenas y colombianas. En ambos contextos su origen es urbano -aunque desde luego con vasos comunicantes hacia el campo y las ruralidades-; la mecha que los prendió definitivamente apunta a mecanismos de expropiación financiera de los ingresos personales y familiares (en el caso chileno el aumento de los pasajes del metro y en el colombiano, una reforma tributaria regresiva para paliar el galopante endeudamiento público); y finalmente, las demandas sociales que se han movilizado desde el inicio y en el transcurso de su trámite político apuntan a impugnar las políticas de neoliberalización y en menor medida, de los proyectos de acumulación de capitales principalmente financieros, que estos sustentan e impulsan.

Dado que en los procesos de acumulación en Chile y Colombia tiende a predominar la extracción de rentas de todo tipo -minería, hidrocarburo, monocultivos, entre otras actividades lícitas e ílicitas- sobre las actividades productivas, la escala urbana en ambos casos ha proporcionado un campo fecundo de privatización, mercantilización y transformación de bienes comunes, servicios 
públicos y otros medios urbanos de subsistencia en activos financieros que son incorporados en los procesos de reproducción de capitales ficticios. Adicionalmente, la producción de ambiente construido en sí (edificios, centros comerciales y vivienda) en o para (megaproyectos energéticos y de transporte situados en el campo) las ciudades chilenas y colombianas, se ha convertido en las últimas décadas en una esfera central para la acumulación de capitales en especial para la reproducción del capital financiero.

En los últimos años el proceso de financiarización de la producción capitalista de ambiente construido es el que más atención teórica, metodológica y empírica ha recibido tanto en el contexto de los urban studies y la urban geography anglosajona como en el ámbito ibérico y latinoamericano. De hecho, lo que Aalbers (2018) ha denominado como 'financiarización urbana' se restringe casi que exclusivamente la subordinación de las lógicas de producción inmobiliaria, de vivienda e incluso de políticas públicas urbanas, a las dinámicas de los mercados de capitales financieros.

Aunque ese sesgo ha facilitado importantes hallazgos sobre los procesos de financiarización capitalista en la escala urbana, acá se parte de la hipótesis de que la 'financiarización urbana' implica una mutación en paralelo en las relaciones sociales de producción y reproducción del espacio urbano, razón por la cual es necesario identificar los elementos centrales sobre los debates en torno a esa categoría en geografía urbana y proponer una agenda teórica que sitúe al mismo nivel de atención la financiarización de los procesos de reproducción social en y mediante los espacios urbanos. Esto último además de facilitar la comprensión de las causas de los estallidos sociales en contextos como el chileno o colombiano, que se pueden conceptualizar como crisis de raíces urbanas' en el sentido harviano del término (Harvey, 2013), favorecería su crítica y la identificación de cambios sociales necesarios para su superación.

En consecuencia, el objetivo del presente artículo es identificar los 'campos ciegos' -vacíos teóricos y áreas de debate- de las discusiones sobre la financiarización urbana y plantear una agenda teórica de carácter exploratorio que gira en torno a la conceptualización de las formas contemporáneas de 'subsunción' de lo urbano a los procesos de reproducción del capital financiero.

Así, en primera instancia, se hará una contextualización crítica sobre los principales modelos de análisis teórico de la financiarización urbana en geografía, haciendo énfasis en la literatura reciente publicada en el ámbito iberoamericano, brasileño y francófono -que es constantemente invisibilizada en las revisiones anglosajonas sobre el tema-; posteriormente se identifican los vacíos teóricos y áreas de contradicción discursiva concebidos como campos ciegos por Lefebvre (1970); en particular el debate entre la financiarización como un proceso de rentismo o de valorización de capital, las dialécticas de la escala que anteponen lo asocian a un fenómeno global pero localmente diferenciado y sobre todo la disociación entre la financiarización de la producción de ambiente construido y la de la reproducción social, asociada a la privatización, mercantilización y conversión activos financieros de los medios urbanos de subsistencia. Finalmente, se esboza una agenda teórica exploratoria sobre las formas de subsunción de lo urbano y de la vida cotidiana en las ciudades a la reproducción del capital financiero. 


\section{Financiarización y producción de ambiente construido: esquemas teóricos de análisis geográfico}

Si bien la financiarización es un término que recibe a veces un uso ligero -en ocasiones se emplea más como una noción ${ }^{3}$ más que como una categoría teórica-, presentando una multiplicidad de significados no necesariamente equivalentes, o incluso siendo cuestionado por su presunta inutilidad heurística (Christophers, 2011), parece haber llegado para quedarse (Barnes \& Christophers 2018). Esto ocurre esencialmente porque los procesos a los que alude tienen especial relevancia para comprender las realidades sociales y espaciales contemporáneas, entre ellas las urbanas. Designa un creciente dominio del capital financiero y sus lógicas de acumulación sobre los ámbitos de la producción y la reproducción social que extiende sus implicaciones más allá de lo económico, hacia lo político y lo cultural (Santana, 2018).

Aunque hay acuerdo sobre sus orígenes espaciales y temporales, situados entre los setenta y ochenta en los grandes mercados financieros de Nueva York y Londres (Chesnais, 2009; Lapavitsas, 2016; Duménil \& Lévy, 2009), no resulta igual cuando se busca explicar el rol que desempeña en los procesos contemporáneos de acumulación de capitales: Sassen (2011) no duda en considerar la financiarización como una nueva forma de capitalismo que trasciende su forma industrial; para Arrighi (1994) quien se refiere más a una 'expansión financiera', es un ciclo geohistórico que se da en la larga duración, anunciando cambios en las hegemonías geopolíticas; finalmente, en el extremo de las posiciones, se sitúan quienes asocian -según Harvey (2019), de manera algo estruendosa- este proceso como parte de un proceso tendencial de descomposición del capitalismo (Kurz, 2016; Jappe, 2016; Wallerstein, 2016).

Hay otra entrada aún menos explorada a dicho proceso, que no excluye elementos de las perspectivas ya mencionadas. Harvey en una entrevista reciente con Albet y Benach (2020), aclarando la importancia de las luchas políticas urbanas en contra de la desposesión como un fenómeno que no es reducible a la explotación por medio de la extracción de plusvalía -de capitalistas a trabajadoras y trabajadores-, plantea que esta "no deja de ser una política de realización de clase porque es una clase la que realiza una extracción: es una clase de rentistas, aunque creo no hemos analizado suficientemente las actividades de los propietarios, de los rentistas, de todos los demás" (Harvey en Albet \& Benach, 2020: 77). De esa manera sugiere que una de las mutaciones del capitalismo contemporáneo, es un creciente dominio de las facciones capitalistas financieras y mercantiles tanto de los procesos de extracción de valor y plusvalía (en el trabajo) como mediante la captación de rentas a partir de fuentes muy diversas sometidas a lógicas de expropiación: "hoy puede decirse que se produce una gran cantidad de valor y plusvalía, pero gran parte de va hacia el lado equivocado o hacia los bancos" (Harvey en Albet \& Benach, 2020: 78).

En ese sentido, la financiarización del capitalismo responde tanto a procesos de dominación financiera en el ámbito de la producción como en el de la reproducción social, aunque el peso de ambos procesos, o la magnitud en la que se combinan, es bastante desigual según el contexto de la acumulación. Ante la persistencia histórica e incluso la creciente preponderancia de formas

Por ejemplo, cuando es reducida a sinónimo de instrumentos como los fondos de inversión, la securitización o la desbancarización del finan ciamiento de empresas. 
rentistas y parasitarias de articulación capitalista como ha ocurrido en Chile, Colombia u otros contextos de América Latina o procesos novedosos de 'capitalismo rentista' que son producto de las últimas décadas inclusive en economías capitalistas potentes como la de Inglaterra -magistralmente analizada por Christophers (2020)-, se producen distintos procesos de acumulación orientadas a la extracción y conversión de rentas en capitales financieros.

En geografía las aproximaciones teóricas que vinculan la financiarización como proceso de cambio capitalista (Carroué, 2011; Méndez, 2018) son más bien escasas. No obstante, en la disciplina se ha avanzado en el análisis teórico de la relación entre financiarización y la producción inmobiliaria, generalmente muy ligadas a experiencias concretas: Suiza con una larga tradición de paraíso fiscal y enclave financiero desregulado, España por su modelo de acumulación basado en la construcción y el turismo que data de las últimas décadas del siglo XX, Estados Unidos -cuna de las Subprime y epicentro de la crisis sistémica de 2008-, Brasil -como la primera potencia capitalista suramericana que experimentara un boom durante la primera década del siglo XXI- o Chile, un caso de temprana implementación de modelos de acumulación financiera desde mediados de los setenta. Ahora bien, algunas provienen del ámbito de la geografía económica mientras que otras han sido impulsadas desde la geografía urbana y más concretamente desde las corrientes críticas o de economía política espacial.

Sin ánimo de exhaustividad se presentan y contrastan a partir de sus aportes para la comprensión de las nuevas formas del desarrollo urbano desigual, cinco aproximaciones que no son siempre excluyentes entre sí y cuya comparación ofrece la identificación de puntos de acuerdo, pero también de diferencias y campos ciegos.

\section{La evaluación financiera, la producción inmobiliaria a 'distancia' y las negociaciones por la ciudad financiarizada}

Una de las aproximaciones seminales en geografía de la financiarización de la producción inmobiliaria es la que proponen Theurillat (2010) y Theurillat et al. (2006; 2015; 2016). Se inspira esencialmente en el caso suizo girando en torno a los fondos de pensiones como agentes claves que median entre el sector financiero y el inmobiliario habitacional. El primer elemento conceptual es que la financiarización se define como un fenómeno de evaluación sistemática de activos por parte de instituciones financieras a partir de tres principios: rentabilidad, liquidez y riesgo (Theurillat, 2010).

Los sistemas de evaluación están integrados por instituciones, circuitos (canales de inversión) con distintas temporalidades y territorialidades según las diferentes perspectivas de rentabilidad, liquidez y riesgo (Theurillat, 2010). La incorporación de lo que Theurillat et al. (2016) denominan 'sistemas inmobiliarios' a esos sistemas de evaluación financiarizados, ha supuesto la introducción de un nuevo circuito capitalista de producción de ambiente construido que coexiste con el más tradicional: en este último caso, dicho circuito se denomina 'real' (Theurillat et al., 2016) o 'directo' (Theurillat, 2010).

El circuito inmobiliario directo o real se caracteriza por las lógicas de 'gestión interna', 'proximidad' y 'evaluación propia' (Theurillat, 2010). La primera alude al establecimiento de relaciones interpersonales que vinculan a las empresas con otros actores públicos y privados mientras que 
la segunda implica un conocimiento profundo de las dinámicas locales del mercado inmobiliario -sean políticas, económicas o sociales-. La tercera es una evaluación establecida dentro del marco mismo de las empresas promotoras y constructoras que se encamina a establecer estrategias temporales y territoriales de maximización de ganancias y minimización de riesgos.

El nuevo circuito de inversión inmobiliaria se caracteriza por su carácter indirecto, es decir, que la gestión tiende a ser externa dado que está en manos de instituciones financieras no necesariamente especializadas en la producción inmobiliaria y trasciende lo local involucrando actores de orden global (Theurillat, 2010); esto implica un proceso de evaluación de activos inmobiliarios que se hace a 'distancia' -usualmente fuera de los mercados inmobiliarios locales y en ausencia de relaciones interpersonales estrechas- lo cual altera la lógica espacial de proximidad más tradicional asociada a la producción inmobiliaria. Finalmente, y dado el carácter financiarizado de las decisiones de inversión inmobiliaria, las estrategias de los actores se orientan no solo al binomio ganancia-riesgo sino al trinomio rentabilidad, liquidez y riesgo (Theurillat, 2010).

Para el caso de Suiza Theurillat (2010) plantea que a pesar de que la toma de decisiones está totalmente concentrada en Zúrich -que es el asiento de fondos de pensiones, sociedades inmobiliarias y fondos de inversión-, las estrategias de inversión favorecen los niveles más altos de la jerarquía urbana del país alcanzando con mucho menor volumen a las medianas y pequeñas ciudades. Esto ha implicado una mayor competencia con el circuito directo, asentado también -pero no exclusivamente- en las grandes ciudades que contribuye a impulsar burbujas de precios de activos inmobiliarios residenciales y comerciales. Ello plantea un dilema en términos de 'sostenibilidad' que el autor achaca directamente y en mayor proporción al circuito indirecto (Theurillat, 2010).

Más recientemente, Theurillat et al. (2016) han construido varios tipos ideales de aterrizajes del capital financiero en la ciudad: 1) la ciudad financiarizada, 2) la ciudad empresarial y 3) la ciudad negociada. Los tres dependen de algo que los autores denominan como 'valor urbano' y que, sin ser definida directamente, parece designar el conjunto de externalidades positivas y negativas que son producto de la construcción colectiva de la ciudad; así cada tipo de anclaje territorial del capital financiero a la ciudad depende de distintas formas de evaluación de tales 'valores urbanos'. En el primer caso, la ciudad financiarizada, dicha apreciación es construida por actores financieros (inversionistas e intermediarios financieros) a distancia a partir de sendos procesos de abstracción cuantitativa que tienden a clasificar activos inmobiliarios (por su nivel de riesgo, liquidez o rentabilidad potencial) desagregados en unidades aisladas (edificios, viviendas, locales) sin ningún contexto socioespacial. Ese proceso de valuación a distancia se asocia con una temporalidad específica: inversiones inmediatas que buscan ganancias a corto plazo, lo cual trae aparejado incursiones efímeras por parte de los inversionistas que pueden acelerar la obsolescencia del ambiente construido (Theurillat et al., 2016).

En la ciudad empresarial, hay una suerte de mediación entre la evaluación financiera a distancia de activos urbanos y las decisiones de inversión, que ejercen coaliciones de crecimiento público-privadas -agencias del estado, empresas promotoras y constructoras locales-. Actúan estas últimas como una suerte de 'traductoras' que favorecen una clasificación más completa y tangible de activos según su valor urbano y las lógicas de proximidad que lo alteran (Theurillat et al., 2016). 
Finalmente, en la ciudad negociada hay supuestamente unas configuraciones más horizontales que involucran a la ciudadanía en los procesos de valuación y que facilitan según Theurillat et al. (2016) que las dimensiones experienciales y emocionales asociadas a distintos lugares, sean incorporadas a la negociación en torno a las rentas urbanas y que haya un 'ganar-ganar' que beneficiaría a los inversionistas, las coaliciones de crecimiento público-privadas y a la ciudadanía en general. Los autores terminan criticando a Harvey por plantear la relación entre capital financiero y producción del espacio urbano como un juego de suma cero.

\section{Del complejo financiero-inmobiliario al cuarto circuito: reescalamiento de la deuda hipotecaria y nuevas economías políticas de la vivienda}

Si desde el punto de vista anterior se cuestiona la perspectiva harviana y se anuncian supuestos efectos positivos de la financiarización de la ciudad mediante la negociación -siendo más una 'cuestión de método-, desde la economía política de la vivienda se ha buscado ir más allá, criticando el proceso y sus implicaciones socioespaciales. En ese sentido los trabajos de Aalbers $(2006 ; 2009 ; 2012 ; 2016)$ ofrecen una serie de conceptos que vinculan la financiarización con la provisión de vivienda y la producción de ambiente construido y el rol del Estado en la financiarización urbana.

Para Aalbers (2016) la financiarización es un patrón de acumulación de capitales que opera mediante canales financieros y no tanto sobre las tradicionales vías de la producción. Esa definición amplia y vinculada al funcionamiento del capitalismo contemporáneo implica según el autor, la necesidad de actualizar y reformular el esquema de los circuitos de acumulación propuesto por Harvey, que según lo que plantea tiene un fuerte aroma keynesiano. Así, la securitización de hipotecas que alcanzó proporciones considerables en mercados financieros de Norte América y Europa durante la primera década fue el principal mecanismo para desplazar sectorial y masivamente, capitales al ambiente construido y a los mercados de vivienda (Aalbers, 2016).

No obstante, el rol de las facciones del capital financiero no es el de simple coordinador de ese desplazamiento sectorial, sino que, según el autor, las finanzas han emergido como un circuito cuaternario de acumulación: estas se constituyen en un mercado para hacer dinero a partir del dinero y como una fuerza independiente que subsume a los demás circuitos a sus propias lógicas (Aalbers, 2016). El movimiento de capitales hacia ese circuito no funciona como en los demás -que compiten por atraer capitales excedentes- sino que este es en sí y para sí, el canal principal de inversión (Aalbers, 2016).

La financiarización de la ciudad es el corolario de ese nuevo circuito cuaternario y de la constitución de lo que el mismo Aalbers (2009) denominó complejo financiero-inmobiliario. En un conjunto de tres reportes sobre la financiarización $(2018 ; 2019 ; 2020)$ y en otras obras de mayor envergadura (Aalbers, 2011; 2012; 2016), el autor ha planteado una serie de tendencias socioespaciales: la primera es la transformación de los activos inmobiliarios, anclados territorialmente, en activos líquidos que se transan mediante distintos instrumentos en los mercados de capitales, que es una idea tomada de los trabajos de Gotham $(2006 ; 2009)$ y la financiarización de promotoras y constructoras (Aalbers, 2019). Sobre este último proceso el autor ha reflexionado poco en sus obras más orientadas a la economía política de la vivienda. 
La segunda es la emergencia de mercados hipotecarios secundarios de operación global que son producto de una mutación escalar de los mercados primarios de hipotecas que eran de índole nacional. Aunque persisten en ciertos contextos las tradicionales prácticas del red lining, la securitización de las deudas hipotecarias ha favorecido un apalancamiento y con ello una mayor extensión social y geográfica de los créditos, con el costo de propiciar formas crediticias predatorias que exponen a una mayor vulnerabilidad a las clases populares. Tras la crisis de 2008 y en diversos países este último proceso se tradujo en unas geografías urbanas marcadas por los desahucios y sendos procesos de acumulación por desposesión en donde proporciones considerables del parque de viviendas terminaron en manos de los bancos (Aalbers, 2012; 2016; Rolnik, 2018).

Una tercera tendencia abordada por Aalbers $(2018 ; 2020)$ tiene que ver con la financiarización de los gobiernos urbanos y del Estado en general. El estado es objeto y agente mismo de la financiarización, es decir su presupuesto y sus mecanismos de financiamiento son insertados en los mercados de capitales -pignoración de partes del presupuesto público, por ejemplo- y a la vez, las administraciones urbanas tienden a incorporarla como estrategia que busca acrecentar su poder político o incluso por obligación, con las consecuentes implementaciones de formas de gobierno corporativo que se imponen a las prácticas de la planeación urbana y a la dirección del sector público en general.

\section{Los nuevos ciclos inmobiliarios y la urbanización imbricados en las 'telarañas financieras'}

Desde la geografía económica y a partir del conspicuo caso español, Méndez, ha planteado análisis mucho más comprehensivos de las geografías de la financiarización (Méndez, 2018) y desde allí ha planteado algunos conceptos para estudiar la relación entre dicho proceso y la producción del espacio urbano (Méndez, 2020). Para el autor la financiarización sería un nuevo régimen de acumulación capitalista que tiene como característica esencial no tanto ser una nueva forma de organización de la producción sino más bien un fenómeno de expansión financiera que implica la imposición de sus lógicas a las demás actividades de la acumulación y la producción de capitales ficticios (Méndez, 2018; 2020).

En primer lugar, las dinámicas de concentración y centralización de capitales financieros en gigantescas instituciones financieras, la densificación de flujos financieros transnacionales, la expansión de la banca en la sombra y de las finanzas offshore, el creciente poder de inversionistas institucionales que alimentan tales flujos y de las instituciones del sector a partir de la imposición de la gobernanza corporativa al resto de los sectores económicos, serían los mecanismos causales de lo que Méndez (2020: 28) denomina como "aumento de la inversión inmobiliaria y de la mercantilización urbana". Este último fenómeno implica unos cambios en el segundo circuito y en los ciclos inmobiliarios, así como nuevas geografías del mercado hipotecario y de lo que denomina 'coaliciones financiero-inmobiliarias' (Méndez, 2018).

El autor parte señalando que el aumento de la inversión inmobiliaria puede estar o no asociada al aumento de la demanda de bienes inmuebles -viviendas, oficinas, locales comerciales, etc.- como lo demuestran los niveles extraordinarios que esta, alcanzó en Norteamérica y Europa Occidental entre fines de los noventa y 2007, para repuntar nuevamente tras la crisis de 2008 , 
desde el año 2013. La financiarización introduciría al esquema tradicional de la producción inmobiliaria capitalista -orientada siempre a la demanda efectiva, más que a la real-, una mayor masa de capitales excedentes en búsqueda de reinversión y consecuentemente una intensificación del carácter especulativo de este tipo de negocios urbanos que desde luego es social y territorialmente muy selectiva (Méndez, 2020).

En términos simples el autor propone un esquema general, aunque inspirado originalmente en el caso español, que plantea el rol central que ejerce el mercado financiero en la dinámica inmobiliaria en la producción -crédito a promotores y constructores- y circulación -crédito hipotecario a hogares- de inmuebles. Aunque el Estado aparece con un rol importante en ambos momentos, Méndez (2020) plantea la posibilidad de su sujeción a los intereses privados de los agentes financieros. De esa manera, la intensificación de los flujos financieros y de la producción de dineros ficticios desembocarían en una ampliación simultanea de la producción y de la circulación inmobiliaria que trae como consecuencia no solo el mayor endeudamiento de empresas y hogares sino una creciente dependencia de las instituciones financieras respecto a otras, para garantizar la liquidez necesaria -como ocurrió entre la banca española y la alemana en la primera década de los dos mil- (Méndez, 2020).

Esa configuración de poder específica que reúne en torno a la gobernanza corporativa a instituciones financieras (que la lideran e imponen), al Estado, a los promotores y constructores entre otros, tiende a conformar un bloque o una coalición que plantea objetivos referidos a las condiciones de posibilidad requeridas para ampliar social y espacialmente, los ámbitos de producción y consumo inmobiliario: neoliberalización del aparato estatal, flexibilización o desregulación de la planeación urbana, privatización y mercantilización de bienes públicos, etc. (Méndez, 2020). Esas estrategias desde luego tienen implicaciones en términos de los ciclos inmobiliarios urbanos y metropolitanos.

Méndez (2020) define un ciclo inmobiliario como el movimiento ascendente y descendente de la actividad y los precios inmobiliarios que tienen una fase de expansión y otra subsecuente de recesión: en la primera predominan procesos de acumulación y valorización del capital invertido en la producción del espacio urbano (además de las burbujas de precios) mientras que en la segunda ocurren procesos de acumulación por desposesión y devaluación del ambiente construido. En esos momentos expansivos ocurren sendas dinámicas de creación y destrucción creativa de las formas urbanas: Méndez (2020) las clasifica según su carácter centrífugo -dispersión urbana y estandarización periférica- y centrípeto -renovación urbana, megaproyectos y revalorización de áreas centrales- pero sostiene que el resultado común es una mayor fragmentación socioespacial.

Sobre las geografías urbanas que emergen de la desvalorización y de los procesos de acumulación por desposesión, Méndez (2020), no plantea un esquema general a pesar de apuntar minuciosamente a las dinámicas de desahucio de la vivienda que ocurrieron en el caso español y que han dado lugar a una abundante bibliografía (Vives-Miró et al., 2018) y a la cual ha aportado el autor en otras obras (Méndez et al., 2015). No obstante, propone un esquema para interpretar las relaciones entre crisis inmobiliaria, financiera y económica que funciona como una suerte de dominó en el que cualquiera de estas puede potencialmente, desencadenar las demás.

A diferencia de los esquemas conceptuales anteriores el que propone Méndez dialoga directamente tanto con los aportes anglosajones y francófonos, así como con los que se han hecho 
en América Latina, especialmente en Chile. Aunque en este último país gran parte de la reflexión sobre las geografías urbanas de la financiarización surgió primero del campo de los estudios urbanos (Daher, 2013; De Mattos, 2007; 2015) en años recientes y a partir de tales aportes, se han elaborado algunas aproximaciones geográficas.

\section{De la financiarización a la metamorfosis metropolitana}

Una primera línea de aproximación a las geografías financieras en Chile es la de Daher (1995a) quien ya en la década de los noventa propuso una suerte de disimetría espacial entre regiones captadoras de fondos bancarios y previsionales, y regiones de colocación (inversión) aunque haciendo un análisis que no aterrizaba -por la naturaleza de los datos- en la escalas urbanas y metropolitanas. En otro trabajo de esa década Daher (1995b) planteó un análisis pionero de los efectos de la creación de los primeros fondos de inversión inmobiliaria en 1991, sosteniendo que tendrían un impacto de desintermediación financiera en la oferta y demanda inmobiliaria y que para su primer cuatrienio mostraban una alta selectividad espacial -concentración en la principal región del país, la Metropolitana y allí en las comunas de mayor ingreso del eje nororiental- y especialización en inmuebles no habitacionales.

No obstante, las reflexiones conceptuales han llegado más tarde en la última década ya bajo el paraguas conceptual de la financiarización. Sin abordar una conceptualización profunda de esta, Daher (2013) la analiza de manera pragmática como una intensificación de flujos transnacionales de capital -fluidez y liquidez como lo plantean los autores de la escuela suiza- que trae como consecuencias territoriales totalmente localizadas, burbujas de precios de activos y la potencialidad de crisis económicas. Lo que sí plantea el autor es que la financiarización ha acentuado la metropolización de la economía con un efecto muy claro de concentración de las finanzas y las inversiones inmobiliarias, que denomina 'metromonopolización financiero-inmobiliaria' (Daher, 2013).

Tres líneas de análisis conceptual en términos de esa metromonopolización financiero-inmobiliaria pueden destacarse de los trabajos de Daher: la acción de los fondos de inversión inmobiliaria en la producción de ambiente construido, la constitución de economías metropolitanas con un predominio macroeconómico de las actividades financieras y la vulnerabilidad territorial a las crisis financieras. Bajo el primer eje, Daher (2013) concluye que la lógica de alta selectividad espacial sigue siendo el principio rector de las estrategias geográficas de los distintos fondos de inversión inmobiliaria aún, cuando estos tipos de instrumentos de inversión hayan aumentado su participación en el ámbito de la producción de ambiente construido.

Sobre el segundo, el autor plantea que la coalescencia de procesos de desindustrialización y de reorientación de parte de la acumulación hacia las commodities (agronegocios, minería) tiene como efecto una hiperconcentración financiera que es de escala metropolitana (Daher, 2016) que finalmente se convierte no solo en un elemento de vulnerabilidad territorial (por el cual el nivel de riesgo aumenta y se generan nuevas formas -riesgo inmobiliario-) y no tanto por condiciones locales -inestabilidades sociales o políticas- sino por la misma naturaleza de la operación de los mecanismos financieros como los fondos de inversión (Daher, 2013).

Hacia finales de la primera década de los dos mil, De Mattos $(2007 ; 2010)$ introdujo el análisis de la financiarización como uno de los mecanismos causales, junto a la globalización y la neoli- 
beralización estatal, de lo que llamó metamorfosis metropolitana que abarca a la vez, tendencias expansivas del tejido urbano a escala metropolitana y una alta fragmentación socioespacial. En un primer momento De Mattos (2006) define la financiarización como un proceso de autonomización del capital financiero, apoyado en la liberalización de los mercados de capitales y flujos transnacionales, así como en las posibilidades que ofrecen las tecnologías digitales de circulación de información.

En ese sentido, la financiarización de la producción inmobiliaria sería una de las cinco tendencias constitutivas de las transformaciones urbanas en América Latina junto a la arquitectura reticular de las empresas que permite dispersar y concentrar ciertas funciones, la precarización por flexibilización y desregulación de los mercado de trabajo, una ampliación del campo metropolitano de externalidades y una uniformización de los paisajes urbanos por la implantación de artefactos de la globalización (De Mattos, 2006).

De Mattos (2007) introdujo en el ámbito latinoamericano los tres principios de orientación de la financiarización señalados por la escuela suiza: rentabilidad, liquidez y riesgo. El autor plantea que esos tres elementos tendrían una expresión espacial que reflejaría la mayor sujeción de las lógicas inmobiliarias a las financieras, aunque no ofreció sustento empírico de ello. El primero en plantear una verificación de esa hipótesis es Cattaneo (2011) quien logra identificar no solamente la selectividad espacial de los fondos de inversión inmobiliaria, dedicados a la provisión habitacional, sino las combinaciones particulares entre rentabilidad y liquidez que ofrecen localizaciones centrales y periurbanas, siendo las primeras más liquidas y menos rentables, mientras que las segundas ofrecen mayores retornos, pero menos liquidez -al menos en el corto o mediano plazo-.

Posteriormente Santana (2018) identificó que esa selectividad espacial y los juegos entre rentabilidad, liquidez y riesgo tienen una expresión según el nivel jerárquico de la red urbana, las características de las economías regionales y las formas que asume la urbanización - metropolización, turistificación- y también que mientras los mecanismos financiarizados de producción inmobiliaria son selectivos territorialmente, los de expansión del consumo mediante la deuda, tienden a una amplia descentralización espacial (Santana, 2020).

Finalmente, Hidalgo et al. (2019) han extendido los argumentos anteriores, priorizando la relación entre rentas del suelo y financiarización. Eludiendo el problema de la relación entre las geografías de la renta del suelo, la circulación del valor en el ambiente construido y los vaivenes de los precios, se han abocado a investigar las estrategias socioespaciales de extracción de rentas inmobiliarias asociadas a la producción inmobiliaria de la naturaleza -desde luego una segunda naturaleza artificial y casi representacional-, que facilita la circulación de ciertos productos inmobiliarios de alto standing-.

\section{Hegemonía financiera, valorización del espacio y escalas de la financiarización}

En el campo de la investigación urbana brasileña, la financiarización ha sido objeto de tratamientos conceptuales y empíricos desde hace también, más de década y media (Volochko, 2008; Fix, 2011; Sanfelici, 2013; Shimbo, 2010). Las reflexiones sobre la financiarización aunque son muy variadas en el ámbito de la geografía como lo demuestra el volumen recién editado por De 
Oliveira et al., 2019, presentan al menos dos tendencias conceptuales, no necesariamente contrapuestas: una más articulada a los planteamientos anglosajones (Sanfelici \& Halbert, 2016), con una atención especial en las escalas del proceso (Sanfelici, 2015) y otra que se desprende de la geografía urbana crítica de la Escuela de Sao Paulo (Volochko, 2008; 2015); Botelho, 2007), que aunque no necesariamente recurra explícitamente a dicha categoría sí elabora planteamientos para comprender la relación actual entre las lógicas del capital financiero y la urbanización (Carlos; 2011; Lencioni, 2014).

Respecto a la última, Carlos (2011) sostiene que el capitalismo se caracteriza actualmente por una hegemonía financiera que tiene hondas implicaciones en la reproducción de las relaciones sociales mediante la producción del espacio urbano: la esencial, es la masificación de la producción del espacio urbano como una totalidad de mercancías inmobiliarias. Tras una crítica a Harvey por reducir la producción capitalista del espacio urbano como simple producción de ambientes construidos, Carlos (2011) retoma el concepto de valorización del espacio -tradicional de la geografía crítica brasileña- para fundamentar un análisis basado en los procesos geográficamente desiguales de valorización -de unidad y oposición entre trabajo muerto y vivo, o entre fijación y movilidad del capital-dinero- en lo urbano. Las discusiones -ya eternas y no siempre concluyentes- que giran exclusivamente sobre la renta del suelo son consideradas por la autora como insuficientes para entender los procesos de mercantilización contemporáneos del espacio urbano.

A partir de ese marco, Volochko (2015) sostiene que la financiarización que actúa tanto en la producción inmobiliaria y la construcción como en la provisión de crédito hipotecario, ha implicado al menos en Brasil nuevas lógicas de valorización del espacio urbano que se caracterizan en términos socioespaciales por la diversificación social y geográfica de las inversiones inmobiliarias, como lo demuestra la penetración por parte de agentes financiero-inmobiliarios del mercado de la vivienda social -en el marco del ya celebre Mi Casa, mi vida- y la apertura de frentes periféricos de producción inmobiliaria, incluidas las áreas tradicionalmente devaluadas, populares y caracterizadas originalmente, por la autoconstrucción.

Ahora bien, además de la estandarización de los paisajes metropolitanos, que el autor asocia a la introducción de técnicas constructivas masivas de bajo costo, aparece como elemento central y contradictorio de las nuevas lógicas de financiarización de la producción inmobiliaria, la necesidad de mantener el localismo de dichas operaciones -conocimiento de mercado local, nexos con los poderes públicos- y de ampliar la diversificación geográfica por parte de las instituciones financieras y de los inversionistas concentradas sobre todo en la gran metrópolis financiera del país o incluso fuera de este. Según Volochko (2015) esto ja implicado una tendencia a la tercerización de la producción inmobiliaria.

Esta contradicción latente entre escalas geográficas contrapuestas de la financiarización y las lógicas inherentes de la producción inmobiliaria ha sido también abordada en detalle por Sanfelici (2015) y constituye uno de los mayores aportes de la geografía brasileña al tema. El autor señala que tanto la financiarización como su operación en los circuitos de la producción inmobiliaria deben ser analizados en términos de las escalas de acumulación de capital en las cuales esta se inserta; además, aunque es la global la más preponderante, las demás escalas, nacionales y locales la reconfiguran permanentemente (Sanfelici, 2015). 
Ese mapa variopinto y cosmopolita de análisis geográficos de la financiarización de la producción inmobiliaria urbana, a pesar de la condición siempre somera y poco justa -con la amplia profundidad con la cual se han planteado originalmente- que imponen este tipo de revisiones, da cuenta tanto de una serie de elementos teóricos claves que deben ser retomados como insumos básicos para reflexionar sobre la subsunción de lo urbano a la reproducción del capital financiero pero también indican una serie de campos ciegos que pueden convertirse en campos abiertos para una agenda teórica atenta a ese fenómeno.

\section{Los campos ciegos de la investigación geográfica sobre la financiarización}

Los campos ciegos son las áreas más problemáticas -dada la ausencia de acuerdos conclusivos sobre distintos aspectos- y también las menos abordadas -donde abundan los silencios y muchas preguntas por formular-. A continuación se reflexiona sobre tres campos ciegos, los primeros asociados áreas problemáticas mientras que el tercero es esencialmente un vacío teórico fundamental: 1) la relación entre la producción y circulación de valor en el ambiente construido versus la extracción o creación de rentas y la generación de capital ficticio; 2) la relación entre extremos escalares -lo global y lo local- versus las mesoescalas -nacionales y regionales-; 3) la reducción de la financiarización a un proceso que afecta únicamente la producción de ambiente construido, pasando por alto las dinámicas urbanas de dominación financiera de la reproducción social, más allá de la vivienda -el tema más tratado-, incluyendo también el ingreso, el consumo cotidiano, la sanidad, la educación y los medios de subsistencia en general.

\section{La financiarización: ¿rentismo a ultranza o forma de valorización?}

Uno de los puntos álgidos sobre el cual no hay consensos, ni en economía política ni en geografía urbana crítica, es sobre si la financiarización, como tendencia constitutiva del capitalismo contemporáneo que implica una expansión de la producción capitalista del espacio, favorece más procesos de extracción y creación de rentas del suelo o sí más bien introduce cambios cuantitativos y cualitativos en los procesos de valorización y desvalorización del espacio. Las perspectivas propuestas por Theurillat, Aalbers o Hidalgo y otras y otros, apuntan a lo primero privilegiando ciertas concepciones: en el caso del autor suizo aparece una especie de dicotomía entre una financiarización agresiva que es claramente extractiva y otra que puede ser negociada y en la cual, las rentas se pueden 'co-crear' y gestionar mediante una 'adecuado anclaje' espacial de las inversiones financieras. La última forma sería más sostenible en opinión del autor.

Aalbers ha puesto el acento sobre la transmutación de la propiedad de la vivienda en activo financiero, lo que implica aceptar que la renta es una forma de capital ficticio como lo plantean Harvey $(1990)$ o Haila $(1988 ; 2012)$. Esta postura resulta clave para comprender las economías políticas de la vivienda y su rol contemporáneo en la sociedad, así como en los procesos de acumulación de capital en general. De hecho, el potencial de la vivienda como capital ficticio es lo que impulsa las tendencias contemporáneas de acumulación por desposesión -privatización del parque de viviendas sociales y de las políticas habitacionales, desahucios, expulsión de inquilinos, etc. - en muchas de las ciudades del mundo. 
En el caso de Hidalgo, se hace énfasis en los procesos de extracción de rentas que permite la producción inmobiliaria financiarizada sea en ámbitos metropolitanos o en áreas de frontera urbana impulsadas por la turistificación. No obstante, gran parte de la reflexión sobre los procesos sociales de extracción tienen que ver con las formas monopólicas de la renta asociadas a condición de sitio específicas, como las costeras, fluviales o lacustres, mientras que otro tipo de rentas que potencialmente se 'crean' por parte de la acción del Estado o incluso de las mismas comunidades, no han sido objeto de análisis.

Son las autoras y autores de la escuela paulista de geografía urbana crítica quienes han cuestionado la centralidad y exclusividad de la categoría de la renta no solo para comprender la producción inmobiliaria capitalista (Carlos, 1994) sino su desempeño bajo la hegemonía financiera o la financiarización (Carlos, 2011). De esa manera, este proceso implica cambios cuantitativos y cualitativos en la producción del espacio urbano como mercancía o producto inmobiliario entre los cuales se sugieren nuevos y masivos procesos de valorización que ocurren en las periferias metropolitanas y en distintos niveles de la red urbana. Esa valorización, aunque está mediada por los requerimientos del capital financiero es una fuente para la reproducción del capitalismo mediante la producción de lo urbano, tal y como lo planteaba Lefebvre (1976).

Este recuento evidencia un campo ciego que persiste en la reflexión en geografía urbana y económica sobre la financiarización y la producción del espacio urbano: en los casos en los que se hace énfasis en el carácter rentístico y en la producción de capitales ficticios se pasa por alto que la construcción sigue siendo una actividad con un alto potencial de generación de plusvalías absolutas -ganancias que derivan de trabajo humano intensivo- y que lo que aparecía como una condición de atraso técnico hace varias décadas, tal y como se interpreta en los trabajos de Topalov (1979), se ha convertido en un atractivo para la valorización de capitales en contextos fuertemente azotados por la desindustrialización.

Por otro lado, desde la perspectiva de la valorización del espacio mediada y hegemonizada por capitales financieros, se omiten procesos claves que tienen que ver con cómo se generan y circulan capitales ficticios que coexisten de manera contradictoria con las dinámicas de producción y circulación del valor. Tanto la formación de plusvalías como la de capitales ficticios (Lefebvre, 1970) son una unidad contradictoria en la producción inmobiliaria financiarizada y sus relaciones no han sido teorizadas con rigor.

\section{El problema de las escalas meso: lo que calla la dialéctica global-local}

En todas las aproximaciones analíticas comentadas se prioriza una suerte de dialéctica entre escalas globales y locales de la financiarización, destacando como rasgo central de la producción inmobiliaria financiarizada el principio de la 'telequinesis', es decir, la de impulsar a grandes distancias estrategias abstractas (clasificación por rentabilidad, liquidez y riesgo) de inversión y producción contraponiendo los centros de decisión financiera y los lugares donde se instalan.

Aunque esa dialéctica, con todas las tensiones descritas en dichos esquemas conceptuales, es un elemento central, hay un vacío en la comprensión de lo que podría considerarse las meso escalas de la financiarización: los niveles nacionales, que siguen siendo los ámbitos en donde 
se constituyen las políticas de mercados de capitales, de liberalización del sector financiero y de constitución de mecanismos normativos y financieros de articulación entre las finanzas y la urbanización, así como los niveles regionales y metropolitanos en donde las intenciones de las facciones de capital financiero-inmobiliaria finalmente se convierten en verdaderas estrategias geopolíticas de producción del espacio urbano -a partir de lógicas de homogeneización, fragmentación y jerarquización de lugares en función de su capacidad portante para la valorización y la realización de capitales ficticios-.

Por ejemplo, en el caso colombiano, la financiarización es un proceso liderado por capitales principalmente nacionales -el Grupo Aval, Bancolombia del Grupo Empresarial Antioqueño y el Grupo Bolívar- y en ciertos casos con áreas regionales de monopolio (un solo banco, con sus fiducias y administradoras de fondos de inversión) domina gran parte de los procesos de producción, circulación y consumo de ambiente construido, como ocurre en Medellín y su región metropolitana en torno a Bancolombia (Santana, 2021) o en Bogotá con el Grupo Aval.

Ese predominio de la dialéctica global-local, además de invisibilizar otras escalas fundamentales de la financiarización, contribuye a petrificar las mismas escalas globales y locales. Las primeras siendo concebidas como un agregado etéreo de agentes e instituciones financieras que actúan al margen de cualquier territorialización y las segundas pasando por alto las verdaderas escalas de actuación de las constructoras y promotoras inmobiliarias, cuya tendencia incluso en América Latina ha sido la de reescalarse -vía procesos de centralización y concentración de capitales- al nivel regional, nacional y en ciertos casos, a la transnacional. También la representación de los agentes de la financiarización de escala global a un comportamiento 'irracional' que se contrapone con una supuesta 'racionalidad' de los locales, es una caricaturización problemática.

\section{La reducción de la financiarización urbana a la producción y de allí a la producción de ambiente construido}

Finalmente, hay una desconexión entre las dinámicas de la financiarización urbana asociadas a la producción -en especial la inmobiliaria- y las vinculadas a la reproducción social. Estas últimas han sido abordadas con bastante atención en el contexto anglosajón mediante aproximaciones postestructuralistas que tienden a deslindar la financiarización del capitalismo como proceso socioespacial de sus manifestaciones epifenómicas en la vida cotidiana, en la producción de subjetividades y la experiencia de los lugares asociados a las finanzas y la deuda; no es de extrañar entonces que estas perspectivas centren menos su atención en la comprensión de las formas que asume la expropiación y desposesión financiera en la escala urbana.

En los terrenos de la geografía urbana crítica y de la economía política espacial la dominación financiera en la esfera de la reproducción social (y sus nexos complejos con la que se da en el ámbito de la producción), con todas las implicaciones políticas que se desprenden de las lógicas de acumulación por desposesión en las ciudades y metrópolis, ha sido un eje de análisis postergado y desarticulado -con excepción de sus implicaciones sobre la vivienda- de todas las propuestas teóricas precedentes. Se podría sostener que al menos el contexto latinoamericano, es urgente convertir este campo ciego en un campo abierto a la exploración teórica. En el siguiente apartado se propone una agenda teórica tendiente a ese fin. 


\section{Las formas de subsunción de lo urbano: a modo de reflexión final}

Una de las aproximaciones metodológicas de Marx, de naturaleza dialéctica, consistía en analizar procesos sociales desde distintos niveles de abstracción (Ollman, 2003). Así, el trabajo humano, como acción objetiva sobre la naturaleza, era subsumido mediante distintas relaciones sociales y modos de producción concretos a lo largo de la historia. De esa manera, la relación entre amo y esclavo/a y posteriormente entre capitalista y trabajador/a podía ser comprendida no solo como relaciones sociales de sujeción estrictamente económicas (extracción de plusvalía) sino como relaciones de dominación que en el primer caso se basaban en la autoridad y la violencia directa mientras que en el segundo incluía, además, formas de violencia por coerción y de carácter simbólico. A la primera la denominó subsunción formal y a la segunda subsunción formal del trabajo (Marx, 1972).

No obstante, en el nivel de la sociedad el capitalismo no solo implica la subsunción formal y real del trabajo, sino que como concepto "anticipa la toma capitalista del mundo social como un proceso global que requiere el dominio y la subordinación de todas sus esferas o sistemas sociales" (Rodríguez et al., 2018: 193). Marx (2017) alude entonces con dicha categoría, a una forma de dominación que trasciende geográficamente el lugar del trabajo al mundo entero y a sus múltiples esferas de lo social. Lo urbano es una de esas esferas o sistemas del mundo social que precisamente por ese movimiento, ha adquirido una extrema importancia práctica (Carlos, 2014).

Lo urbano y la ciudad que como procesos sociales y espaciales se articula mediante la centralidad y simultaneidad (Lefebvre, 1976), son unas de las fronteras clave sobre las cuales se han expandido nuevas 'formas de subsunción' vinculadas a las lógicas del capitalismo financiero y rentista. Se podrían entonces plantear de manera muy somero, cuatro tesis de exploración teórica y práctica:

1) La subsunción financiera implica un dominio creciente sobre los procesos de producción y reproducción social de lo urbano. Tanto la producción de valor como el acceso a los medios urbanos de subsistencia están sujetos a los principios de reproducción del capital financiero: predominio de liquidez sobre el riesgo, rentabilidad construida sin mayor asociación con las dinámicas de valorización del capital y necesidad de incremento cuantitativo y diferenciación cualitativa y socioespacial de fuentes de extracción de rentas.

2) La escala de lo urbano, como ámbito para la acumulación, asume un rol neurálgico por dos circunstancias: por un lado, la ciudad es un reservorio amplio y diverso de bienes comunes y públicos susceptibles de ser transformados en activos financieros y en fuentes de extracción de rentas; por otro, la producción de ambiente construido favorece la generación de procesos de valorización (escasos o inexistencias en otras ramas capitalistas) y a la vez de segmentación de los productos inmobiliarios y las infraestructuras como activos financieros.

3) La subsunción financiera se construye continuamente mediante prácticas de acumulación por desposesión que se dan bajo estrategias socioespaciales de expolio (robo mediante violencias físicas) y expropiación (robo mediante violencias simbólicas y jurídicas). 
4) Se reproduce mediante la sujeción e internalización de los sujetos sociales a los mecanismos de la deuda, del consumo privado e individual, de la competencia feroz por subsidios y desde luego mediante instrumentos coacción (amenaza de desposesión) o coerción (ejercicio de la violencia estatal, por ejemplo). La vida cotidiana en lo urbano se subsume así a la reproducción financiara del capitalismo.

El resultado de esas formas de subsunción de lo urbano es lo que Merrifield (2020: 189) denomina modo de urbanización parasitario cuya expresión material es la de la ciudad parasitaria:

“¿De quién es la ciudad? La respuesta quizá esté bastante clara: la de los parásitos y su descendencia es una especie que podemos etiquetar como ciudad parasitaria [ ] los parásitos devoran la riqueza común del mundo, devoran el cuerpo social desde dentro, despojan a la gente de sus bienes, embargan sus casas, despojan de valor, en vez de contribuir a generarlo. En las ciudades parasitarias, la riqueza social por empresas ostentosamente ineficientes administradas por élites parasitaria, por nuestra propia aristocracia (el 1\%) que derrocha capacidad generativa al prosperar a partir de actividades improductivas"

Las crisis urbanas en Chile y Colombia son una respuesta precisamente a ese modo de urbanización y por ello las distintas formas de subsunción de lo urbano a la reproducción del capital financiero explican la magnitud de la resistencia, la amplia diferenciación social de la misma y la virulencia en las respuestas del Estado. La máxima de Hilferding se cumple a cabalidad: "el capital financiero no quiere la libertad, sino la dominación" (citado en Merrifield, 2020: 201).

\section{Referencias}

AALBERS, M. Geographies of the financial crisis. Area, 2009, N 41, Vol. 1, p. 34-42.

AALBERS, M. Place, Exclusion and Mortgage Markets. Londres: Wiley Blackwell, 2011.

AALBERS, M. Subprime cities. The Political Economy of Mortgage Markets. Londres: Wiley Blackwell, 2012.

AALBERS, M. The Financialization of Housing: A political economy approach. Londres: Routledge, 2016.

AALBERS, M. Financial geography I: Geographies of tax. Progress in Human Geography, 2018, Vol. $42, N^{\circ} 6$, p. 916-927.

AALBERS, M. Financial geography II: Financial geographies of housing and real estate. Progress in Human Geography, 2019, Vol. 43, N² 2, p. 376-387.

AALBERS, M. Financial geography III: The financialization of the city. Progress in Human Geography, 2020, Vol. 44, N³, p. 595-607. 
ALBET, A. \& BENACH, N. Espacio, tiempo y capital. Una conversación con David Harvey. En BENACH N. \& ALBET A., David Harvey. La lógica geográfica del capitalismo. Barcelona: Icaria, 2009, p. 59-100.

ARRIGHI, G. El largo siglo XX. Madrid: Akal, 1994.

BARNES, T. \& CHRISTOPHERS, B. Economic Geography. A critical introduction. Oxford: Wiley Blackwell, 2018.

CARLOS, A. A Condição Espacial. São Paulo: Contexto, 2011.

CARROUÉ, L. La planète financière; Capital, pouvoirs, espace et territoires. Paris: Armand Colin, 2015.

CATTANEO, R. Los fondos de inversión inmobiliaria y la producción privada de vivienda en Santiago de Chile: ¿Un nuevo paso hacia la financiarización de la ciudad? EURE, 2011, Vol. 37, № 112, p. 5-22. http://dx.doi.org/10.4067/S0250-71612011000300001

CHESNAIS, F. La preminencia de las finanzas en el capitalismo "en general", el capital ficticio y el movimiento contemporáneo de mundialización del capital. En: DE BRUNHOFF S., CHESNAIS F., DUMÉNIL G., HUSSON, M. \& LÉVY, D (eds.), Las finanzas capitalistas. Para comprender la crisis mundial. Buenos Aires: Herramienta ediciones, 2009, p. 84-144.

CHRISTOPHERS, B. The limits to financialization. Dialogues in Human Geography, 2015, Vol. 5, № $2,183-200$.

CHRISTOPHERS, B. Rentier capitalism. Who owns the economy and who pays for it? Londres: Verso, 2020.

DAHER, A. Efecto concentrador de los fondos inmobiliarios. Revista de Ciencias Sociales de la Universidad de Costa Rica, 1995a, № 68, p. 17-32.

DAHER, A. Territorios del capital bancario y previsional en Chile. Documents d'Anàlisi Geogràfica, 1995b, N²7, p. 37-55.

DAHER, A. Territorios de la financierización y de las crisis inmobiliarias. Revista de Geografía Norte Grande, 2013, N 56, p. 7-30.

DAHER, A. De cómo Chicago transformó a Santiago: 40 años de gobernanza de mercado. En A. ORELLANA, F. LINK \& J. NOYOLA (eds.), Urbanización planetaria y la reconstrucción de la ciudad. Santiago de Chile: Colección Estudios Urbanos-RiL Editores, 2016, pp. 141-168.

DE MATTOS, C. Modernización capitalista y transformación metropolitana en América Latina: cinco tendencias constitutivas. En: GERAIGES A., ARROYO M. \& SILVEIRA, M. (eds.), América Latina: cidade, campo e turismo. Sao Paulo: CLACSO, 2006, p. 39-73. 
DE MATTOS, C. Globalización, negocios inmobiliarios y transformación urbana. Nueva Sociedad, $N^{\circ} 212,2007$, p. 82-96.

DE MATTOS, C. Globalización y metamorfosis urbana en América Latina. Quito: OLACCHI-MDMQ, 2010.

DE MATTOS, C. Revolución urbana. Estado, mercado y capital en América Latina. Santiago: RiL-Colección estudios urbanos UC, 2015.

DUMÉNIL, G., \& LÉVY, D. (2009). Las finanzas capitalistas: relaciones de producción y relaciones de clase. En: DE BRUNHOFF S., CHESNAIS F., DUMÉNIL G., HUSSON M. \& LÉVY D. (eds.), Las finanzas capitalistas. Para comprender la crisis mundial. Buenos Aires: Herramienta ediciones, 2009, p. 153-203.

FIX, M. Financiarização e transformações recentes no circuito imobiliário no Brasil. Tesis de doctorado, UNICAMP, Campinas, 2011.

GOTHAM, K. F. Secondary Circuit of Capital Reconsidered: Globalization and the U.S. Real Estate Sector. American Journal of Sociology, 2006, № 112, Vol. 1, p. 235-275.

HAILA, A. Land as a financial asset: The theory of rent as a mirror of economic transformation. Antipode, 1988, N²2, vol. 2, p. 79-101.

HARVEY, D. Urbanismo y desigualdad social. Madrid: Siglo XXI Editores, 1978.

HARVEY, D. The Limits to Capital. Oxford: Basil Blackwell, 1982.

HARVEY, D. The Urbanization of Capital. Studies on History and Theory of Capitalist Urbanization. Oxford: Basil Blackwell, 1985.

HARVEY, D. The urban experience. Baltimore: Johns Hopkins University Press, 1989.

HARVEY, D. Los límites del capitalismo y la teoría marxista. México: Fondo de Cultura Económica, 1990.

HARVEY, D. Towards a Theory of Geographical Uneven Development. Londres: Verso, 2006.

HARVEY, D. Espacios del capital. Hacia una geografía crítica. Madrid: Akal, 2007.

HARVEY, D. París, capital de la modernidad. Madrid: Akal, 2008.

HARVEY, D. Ciudades rebeldes. Del derecho a la ciudad a la revolución urbana. Madrid: Akal, 2013.

HARVEY, D. Guía de El Capital. Libro Primero. Madrid: Akal, 2014. 
HARVEY, D. Senderos del mundo. Madrid: Akal, 2018.

HARVEY, D. Marx, El capital y la locura de la razón económica. Madrid: Akal, 2019.

HIDALGO, R.; ALVARADO, V. \& SANTANA, D. Geografías financiarizadas del extractivismo inmobiliario: lógicas financiero-inmobiliarias y estatales de la producción de lo urbano y la naturaleza en Chile. Em: GODINHO F., DIAS, L., TUNES, R. \& MORAES, R. (orgs,), Espaço e economia. Geografia ecônomica e a economia política. Rio de Janeiro: Consequencia, 2019, p. 385-396.

HILFERDING, R. El capital financiero. La Habana: Instituto Cubano del Libro, 1971.

JAPPE, A. Las aventuras de la mercancía. Madrid: Pepitas de calabaza, 2016.

KURZ, R. El colapso de la modernización. Del derrumbe del socialismo de cuartel a la crisis de la economía liberal. Buenos Aires: Marat, 2016.

LAPAVITSAS, C. Beneficios sin producción. Cómo las finanzas nos explotan. Madrid: Traficantes de sueños, 2016.

LEFEBVRE, H. La revolución urbana. Madrid: Alianza Editorial, 1980.

LEFEBVRE, H. Espacio y política. El derecho a la ciudad II. Barcelona: Ediciones Península, 1976.

LENCIONI, S. Reestruturação imobiliária: uma análise dos processos de concentração e centralização do capital no setor imobiliário. EURE, 2014, Vol. 40, N 120, p. 29-47.

MARX, K. Teorías sobre la plusvalía. Buenos Aires: Cartago, 1972.

MARX, K. El Capital. Tomo I. Madrid: Akal, 2017.

MARX, K. Elementos fundamentales para la crítica de la economía política (Grundrisse) 1857-1858. 3 vols. México: Siglo XXI, 2014.

MÉNDEZ, R, ABAD, L. \& ECHAVES, C. Atlas de la crisis. Impactos socioeconómicos y vulnerabilidad en España. Valencia: Tirant Lo Blanch, 2015.

MÉNDEZ, R. La telaraña financiera. Una geografía de la financiarización y su crisis. Santiago de Chile: Ril-Colección de Estudios Urbanos UC, 2018.

MÉNDEZ, R. Ciudades en venta. Estrategias financieras y nuevo ciclo inmobiliario en España. Valencia: PUV, 2020.

MERRIFIELD, A. La nueva cuestión urbana. Pamplona: Katakrak, 2020. 
OLLMAN, B. Dance of the Dialectics. Steps in Marx's Method. Chicago: University of Illinois Press, 2003.

RODRÍGUEZ, N; PESANTEZ, C. \& RIBADENEIRA, F. Las categorías de subsunción formal y real en Marx: una breve aproximación para comprender la universalidad en el siglo XXI. Revista Economía, 2018, Vol. 70, No 111, p. 187-200.

SANFELICI, D. Financeirização e a produção do espaço urbano no Brasil: uma contribuição ao debate. EURE, 2013, Vol. 39, No 118, p. 27-46. http://dx.doi.org/10.4067/S0250-716120130003000

SANFELICI, D. \& HALBERT, L. Financial markets, developers and the geographies of housing in Brazil: A supply-side account. Urban Studies, 2016, N 53, Vol. 7, p. 1465-1485.

SANFELICI, D. As escalas da acumulação na produção das cidades. En: A. CARLOS, D. VOLOCHKO e I. PINTO (orgs.), A cidade como negócio. São Paulo: Contexto: 2015, p. 121-140.

SANTANA, D. Geografías de la acumulación por urbanización en Chile (1975-2015): ¿utopías de vivienda, distopías urbanas? Tesis de doctorado, Instituto de Geografía, Pontificia Universidad Católica de Chile, 2018.

SANTANA, D. Geografías regionales y metropolitanas de la financiarización habitacional en Chile (1982-2015): ¿entre el sueño de la vivienda y la pesadilla de la deuda? EURE, 2020, Vol. 46, № 139, 163-188.

SANTANA, D. ¡Manos Sobre La Ciudad! Hacia Nuevas Geopolíticas De La Producción De Ambiente Construido en Medellín (2004-2019). ACME, 2021, Vol. 21, Nº 1, p. 34-57.

SASSEN, S. Expulsiones. Brutalidad y complejidad en la economía global. Buenos Aires: Katz Editores, 2015.

SHIMBO, L. Habitação social, habitação de mercado: a confluência entre Estado, empresas construtoras e capital financeiro. Tesis de Doctorado en Arquitectura y Urbanismo, Universidad de São Paulo, 2010.

THEURILLAT, T.; CORPATUAUX; J. \& CREVOSIER. O. The Financialization of the Property Sector: the Case of the Swiss Pension Trusts (1994-2005). Ponencia presentada en el 46 Congress of the European Regional Science Association: "Enlargement, Southern Europe and the Mediterranean", Agosto 30 a septiembre 3, Volos, Grecia, 2006.

THEURILLAT, T. La financiarisation de l'inmmobilier et la durabilité. Le cas des investissements immobiliers des caisses de pensión suisses. Saarbrücken: EUE, 2010.

THEURILLAT, T, RÉRAT, P. \& CREVOSIER, O. The real estate markets: Players, institutions and territories. Urban Studies, 2015, N 52, Vol. 8, p. 1414-1433. 
THEURILLAT, T.; VERA-BÜCHEL, N. \& CREVOSIER, O. Commentary: From capital landing to urban anchoring: The negotiated city. Urban Studies, 2016, N 53, Vol. 7, 1509-1518.

TOPALOV, C. La urbanización capitalista: algunos elementos para su análisis. México: Edicol, 1979.

VIVES-MIRÓ, S., SALAMANCA, O. \& PÉREZ, J. Geografies de la despossesió d'habitatge a través de la crisi: els desnonaments Marca Palma. Barcelona: Icaria, 2018.

VOLOCHKO, D. A produção do espaço e as estratégias reprodutivas do capital: negócios imobiliários e financeiros em São Paulo. Tesis de Maestría em Geografía Humana, Universidad de São Paulo, 2008.

VOLOCHKO, D. A moradia como negócio e a valorização do espaço urbano metropolitano. En: A. CARLOS, D. VOLOCHKO e I. PINTO (orgs.), A cidade como negócio. São Paulo: Contexto, 2015, p. 97-120.

WALLERSTEIN, I. La crisis estructural, o por qué los capitalistas ya no encuentran gratificante el capitalismo. En: WALLERSTEIN, I. COLLINS, R., MANN, M, DERLUGUIAN, G. \& CALHOUN, C. (eds.), ¿Tiene futuro el capitalismo?. México: Siglo XXI, 2016, p. 15-46. 